Abstract 723 Table 1 Comparison of the performance of ROCK-I and ROMA

\begin{tabular}{|c|c|c|c|c|c|c|c|}
\hline & $\begin{array}{l}\text { Sensitivity } \\
(95 \% \mathrm{CI}), \%\end{array}$ & $\begin{array}{l}\text { Specificity } \\
(95 \% \text { Cl), } \%\end{array}$ & $\begin{array}{l}\text { PPV } \\
(95 \% \text { CI), \% }\end{array}$ & $\begin{array}{l}\text { NPV } \\
(95 \% \mathrm{Cl}), \%\end{array}$ & $\begin{array}{l}\text { Accuracy } \\
(95 \% \mathrm{Cl}), \%\end{array}$ & $\mathrm{LR}+$ & $\begin{array}{l}\text { ROC-AUC } \\
(95 \% \text { CI), } \%\end{array}$ \\
\hline ROCK-I & $\begin{array}{l}89.6 \\
(75.3-96.0)\end{array}$ & $\begin{array}{l}93.7^{*} \\
(90-96.1)\end{array}$ & $\begin{array}{l}66.7^{*} \\
(52.4-78.5)\end{array}$ & $\begin{array}{l}98.5 \\
(96-99.4)\end{array}$ & $\begin{array}{l}93.2^{*} \\
(89.7-95.6)\end{array}$ & 14.3 & $\begin{array}{l}0.97 \\
(0.948-0.992)\end{array}$ \\
\hline ROMA & $\begin{array}{l}83.9 \\
(68.5-92.6)\end{array}$ & $\begin{array}{l}85.7^{*} \\
(80.8-89.5)\end{array}$ & $\begin{array}{l}45.2^{*} \\
(33.7-57.2)\end{array}$ & $\begin{array}{l}97.4 \\
(94.4-98.8)\end{array}$ & $\begin{array}{l}85.5^{*} \\
(80.9-89.1)\end{array}$ & 5.9 & $\begin{array}{l}0.94 \\
(0.899-0.981)\end{array}$ \\
\hline
\end{tabular}

ovarian diseases. The superiority of well-known Risk of Ovarian Malignancy Algorithm (ROMA), based on both HE4 and Carbohidrate Antigene 125 (CA125), over CA125 alone and its clinical significance are still debated.

The aim of this study was to elaborate a new algorithm, based on serum CA125, HE4 and age, to assess the risk of malignancy in premenopausal patients with pelvic mass.

Methodology The training dataset included 284 premenopausal patients operated because of presence of pelvic mass, out of which 35 and 249 had malignant and benign disease respectively. A new algorithm based on serum HE4, CA125 and age as variables has been developed by using the scenario of

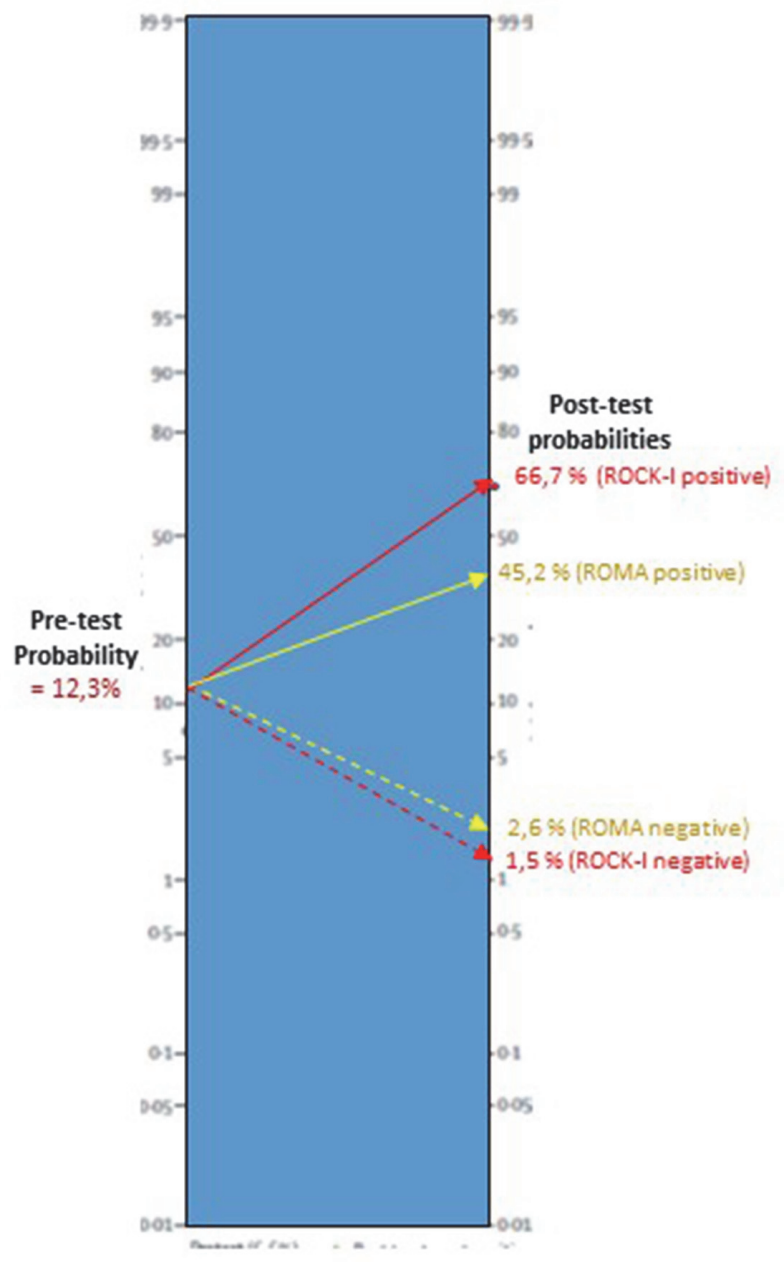

Abstract 723 Figure 1 Pre-test and post-test probabilities of Epithelial ovarian cancer (all stage) together with borderline ovarian tumours stage Ic2-IIIC discrimination "benign disease versus epithelial ovarian cancer (all stages) together with borderline ovarian tumors FIGO stage 1c2-3c". This new algorithm, named Risk of Ovarian Cancer Kazan Index (ROCK-I), was further compared with ROMA and CA125 alone. A recruitment of a validating dataset is presently being concluded.

Result(s)* The original ROCK-I has demonstrated greater diagnostic performance than ROMA (table 1, figure 1). When the above-mentioned scenario of discrimination was used the specificities $(95 \%$ confidence interval (CI)) of ROCK-I and ROMA were 93,7\% (90 - 96,1\%) and 85,7\% (80.8 - 89.5\%) respectively, while the sensitivities $(95 \% \mathrm{CI})$ were $89.6 \%(75.3$ - 96.0) and $83.95(68.5$ - 92.6) respectively and accuracies (95\%CI) were 93.2\% (89.7 - 95.6) and 85.5\% (80.9 - 89.1). The superiority of ROCK-I in specificity, accuracy and positive predictive value was statistically significant $(p<0.05)$, while the tendency towards the superiority in sensitivity and negative predictive value does not reach the statistical significance. Areas under receiver-operating-characteristic curves (95\%CI) of ROCK-I and ROMA were 0.97 (0.948 - 0.992) and 0.94 $(0.899-0.981)$ respectively.

Conclusion* The proposed ROCK-I has demonstrated greater diagnostic performance than ROMA in the analyzed dataset. If an external validation can show similar or even slightly lower difference between ROCK-I and ROMA it may provide a new basis of routine-use of HE4 in patients with pelvic mass.

\section{DISEASE PROGRESSION IN PATIENTS WITH OVARIAN CANCER WHO RECEIVED FIRST-LINE MAINTENANCE THERAPY OR ACTIVE SURVEILLANCE, A US REAL- WORLD ANALYSIS}

${ }^{1} \mathrm{D}$ Chase*, ${ }^{2} \mathrm{JA}$ Perhanidis, ${ }^{2} \mathrm{D}$ Gupta, ${ }^{3} \mathrm{~L}$ Kalilani, ${ }^{4} \mathrm{~T}$ Woodward, ${ }^{5} \mathrm{~A}$ Gonzaléz-Martín. ${ }^{1}$ Arizona Oncology (US Oncology Network), University of Arizona College of Medicine, Phoenix, AZ, USA; ${ }^{2}$ GlaxoSmithKline, Waltham, MA, USA; ${ }^{3}$ GlaxoSmithKline, Durham, NC, USA; ${ }^{4}$ GlaxoSmithKline, Philadelphia, PA, USA; ${ }^{5}$ Clinica Universidad de Navarra, Madrid, Spain

\subsection{6/ijgc-2021-ESGO.455}

Introduction/Background* Although most patients with ovarian cancer $(\mathrm{OC})$ respond to first-line $(1 \mathrm{~L})$ treatment, $\approx 70 \%$ experience recurrence within 3 years. Limited evidence exists on the interaction of prognostic factors and the risk of progressive disease (PD). This study assessed whether the number of risk factors (RFs) impacted time to $\mathrm{PD}$ in patients treated with maintenance therapy (MT) or active surveillance (AS) after completing $1 \mathrm{~L}$ therapy.

Methodology This retrospective cohort study included patients diagnosed with OC between January 1, 2011, and February 
Abstract 724 Table 1 Median time to next treatment by number of risk factors $(95 \% \mathrm{Cl})$, months

\begin{tabular}{|c|c|c|c|c|c|c|}
\hline \multirow{2}{*}{$\begin{array}{c}\text { Type of } \\
\text { treatment }\end{array}$} & \multirow{2}{*}{$\begin{array}{c}\text { High-risk category } \\
(\mathbf{1} \text { risk factor })\end{array}$} & $\mathbf{0}$ & $\mathbf{1}$ & $\mathbf{2}$ & $\mathbf{3}$ & $\mathbf{4}$ \\
\hline \multirow{2}{*}{ MT } & 13.3 & 28.8 & 19.3 & 17.8 & 10.6 & 6.7 \\
& $(11.1-16.3)$ & $(10.8-\mathrm{NA})$ & $(13.5-30.6)$ & $(12.8-22.5)$ & $(7.0-13.4)$ & $(6.2-8.4)$ \\
\hline \multirow{2}{*}{ AS } & 9.0 & 26.4 & 17.7 & 11.3 & 6.5 & 4.0 \\
& $(8.0-9.9)$ & $(14.7-47.8)$ & $(13.4-23.3)$ & $(9.2-12.5)$ & $(5.8-7.7)$ & $(3.5-4.4)$ \\
\hline AS, active surveillance; Cl, confidence interval; MT, maintenance therapy; NA, not achieved. \\
\hline
\end{tabular}

28, 2021, from the Flatiron Health electronic health recordderived de-identified US database. Patients $\geq 18$ years old, with stage III/IV disease, who received $1 \mathrm{~L}$ platinum-based therapy, had ECOG performance score of $0 / 1$, and had $\geq 12$ weeks of follow-up after $1 \mathrm{~L}$ treatment were included. Patients were classified into 2 risk categories: moderate risk (stage III disease, no visual residual disease, primary debulking surgery, and BRCA mutant) or high risk (presence of $\geq 1$ of the following: stage IV disease, visible residual disease, interval debulking surgery/no surgery, or BRCA wild type/unknown status). High-risk patients were further grouped by total number of RFs. Patients were followed from index date (ID), defined as end of $1 \mathrm{~L}$ treatment. The target trial emulation method was used to account for potential selection and immortal time biases. Patients were classified as having received MT if MT was started within 120 days of ID.

Result(s)* Of the 1251 patients with advanced OC evaluated, 26\% $(n=323)$ initiated $1 \mathrm{~L} \mathrm{MT}$ and 74\% $(n=928)$ did not. Of patients who received MT, 5\% $(n=16)$ were moderate risk and $95 \%(n=307)$ were high risk. Only 4\% $(n=34)$ of AS patients were moderate risk and $96 \%(n=894)$ were high risk. Time to next treatment (TTNT) decreased with more RFs (table 1). Notably, among patients in the high-risk category, median TTNT was longer in patients who received 1L MT than in those who received AS (table 1).

Conclusion* The number of RFs impacted the risk of PD, irrespective of the type of treatment the patient received after completion of $1 \mathrm{~L}$ treatment. For high-risk patients, greater TTNT prolongation was associated with 1L MT but not AS.

\section{HRD SCORING WITH A SNP ASSAY}

T Mckee*, Y Christinat, S Leboube, L Ho. Hôpitaux Universitaires de Genève (HUG), Genève, Switzerland

\subsection{6/ijgc-2021-ESG0.456}

Introduction/Background* Homologous recombination deficiency (HRD) is a well-known characteristic of BRCA-mutated tumors, an alteration present in $\sim 30 \%$ of ovarian cancers and with different frequencies in breast, prostate and pancreatic cancers. The PAOLA-1 trial demonstrated that responders to Poly (ADP-ribose) polymerase (PARP) inhibitors do not only include BRCA-mutated tumors but also tumors that are BRCAwildtype and display an HRD phenotype. Given its importance, many different scores and technologies have been proposed and commercialized to assess the HRD phenotype. Nonetheless many of these solutions are expensive and/or complicated to implement in practice.

Methodology We propose a novel HRD scoring algorithm based copy number alterations obtained from a clinical-grade
SNP assay that works on FFPE samples (ThermoFisher Oncoscan Assay). The method has been evaluated on 400 highgrade ovarian carcinoma and 100 triple-negative breast cancer samples from the TCGA cohort. A validation was performed on an internal cohort of 50 ovarian cancers.

Result(s)* The algorithm performed better than two wellknown commercial methods, the compound score from Telli et al. (LST+LOH+TAI) and the percentage of $\mathrm{LOH}$ bases across the genome, and classified correctly the BRCA-mutated cancers into the HRD category.

Conclusion* The high concordance with the LST+LOH+TAI score led to its inclusion into the clinical routine at the Geneva University Hospitals. The method is also being evaluated as a biomarker to predict response to Olaparib as part of phase 3 of the ENGOT HRD European Initiative (EHEI).

\section{THE IMPACT OF AGE AND PERFORMANCE STATUS ON THE MANAGEMENT OF PATIENTS WITH OVARIAN CANCER: A MULTICENTRIC STUDY}

${ }^{1} \mathrm{ME}$ Laudani ${ }^{*},{ }^{2} \mathrm{~L}$ Fuso, ${ }^{1} \mathrm{M}$ Barboni, ${ }^{1} \mathrm{G}$ Parpinel, ${ }^{1} \mathrm{E}$ Peirano, ${ }^{2} \mathrm{M}$ Villa, ${ }^{3} \mathrm{~F}$ Moro, ${ }^{2} \mathrm{~A}$ Ferrero, ${ }^{1} \mathrm{P}$ Zola. ${ }^{1}$ University of Turin, Surgical Sciences, Torino, Italy; ${ }^{2} \mathrm{AO}$ Ordine Mauriziano, Gynecology and Obstetrics, Turin, Italy; ${ }^{3}$ Città della Salute e della Scienza di Torino, Turin, Italy

\subsection{6/ijgc-2021-ESG0.457}

Introduction/Background* Incidence of ovarian cancer increases with age and reaches a peak at 70 years. The aim of the present study is the analysis of therapeutic differences, both surgical and pharmacological, in patients affected by ovarian cancer and stratified by age.

Methodology A multicentre retrospective study has been conducted. Patients with ovarian cancer were included and ranked at diagnosis in group A ( $\geq 70$ years) and group B ( $<70$ years). Co-morbidities, performance status, FIGO stage, grading, histotype and treatment were considered. Surgical treatment was considered as primary debulking surgery or interval debulking surgery (IDS) reporting extension, residual tumour, complications and days of hospitalization. Chemotherapy was administered for 3 or more cycles and the clinical response was evaluated.

Result(s)* 459 patients were included in the study, 132 $(28.8 \%)$ in group A and $327(71.2 \%)$ in group B. Considering advanced stages optimal cytoreduction was achieved in $76.7 \%$ of younger patients and in $66.7 \%$ of older $(p=0.05)$. IDS was especially necessary in in the elderly compared to the young ones $(37.9 \%$ vs $49 \% ; p=0.05)$. There was good correlation between overall survival and the performance status $(p=0.0001)$, the age at diagnosis $(p=0.001)$, tumour stage $(\mathrm{p}=0.0001)$ and residual tumour $(\mathrm{p}=0.00001)$. 